\title{
Vertebrobasilar transient ischemic attacks - a challenging diagnosis
}

\author{
Ionut-Flavius Bratu1, Athena Cristina Ribigan ${ }^{1,2}$, Raluca Stefania Badea1, \\ Florina Anca Antochi', Ovidiu-Alexandru Bajenaru ${ }^{1,2}$ \\ ${ }^{1}$ Department of Neurology, Emergency University Hospital, Bucharest, Romania \\ 2"Carol Davila" University of Medicine and Pharmacy, Bucharest, Romania
}

\begin{abstract}
Objectives. Transient ischemic attacks (TIAs) can present with a large variety of clinical features that impose an extensive differential diagnosis. We report this case due to its particularities of clinical presentation and diagnosis algorithm.

Material and methods. A 72-year old male patient presented to our Neurology Department for recurrent episodes of numbness felt in the right upper limb. The initial patient history deemed the episodes as consistent with simple focal seizures. The cerebral computed tomography scan showed no recent lesions. Furthermore, video-electroencephalography was performed, but revealed no abnormalities. A second, more thorough anamnesis revealed that paresthesia was also present in the left part of the upper and lower lips and that the numbness episodes were being accompanied by blurred vision, loss of balance and difficulties coordinating his right upper limb.

Results. The time-of-flight magnetic resonance angiography revealed dolichoectasia of the left vertebral and basilar arteries. Furthermore, the basilar artery grooved and displaced the pons with respect to the midline. The angio-computed tomography scan of the supra-aortic trunks showed multiple stenoses located in the V4 segment of both vertebral arteries, in the intracavernous segment of the right internal carotid artery and in the proximal segment (90\%) and the middle third part $(80 \%)$ of the basilar artery. The patient was diagnosed with vertebrobasilar TIAs and started treatment with Clopidogrel with no recurrence of the TIAs.

Conclusions. This case was chosen to be presented in order to highlight another facet of the clinical polymorphism exhibited by transient ischemic attacks.
\end{abstract}

Keywords: vertebrobasilar, transient ischemic attack

\section{INTRODUCTION}

The transient ischemic attack (TIa ) represents a short episode of neurologic dysfunction due to focal brain, spinal cord or retinal ischemia, in the absence of evidence regarding acute tissue infarction (1).

In terms of etiopathogenesis, TIa s can be divided into three main subtypes regarding the mechanism underlying the locally decreased blood

(2): embolic TIa s, small penetrating vessel TIa s and low TIAs. Embolic TIAs often consist in single, hour-long episodes of focal neurologic symptoms with the emboli being arterial in origin (usually from an extracranial artery) or of cardiac origin - e.g. in the case of atrial

Small penetrating vessel TIa s are caused by transient cerebral ischemia induced by stenosis of the penetrating arteries originating in the middle cerebral, basilar and vertebral arteries, as well as in the circle of Willis itself, with the stenotic lesion being atherothrombotic and obstructing the origin of the penetrating vessel or of lipohyalinotic origin and being present distally within the penetrating vessel (3). Low TIAs are caused by an abrupt reduction of blood in a vascular territory exhibiting se vere stenotic lesions (very often of atherosclerotic origin). The most common sites associated with TIAs are the origin or the siphon of the internal carotid artery, the stem of the middle cerebral artery and the vertebrobasilar junction (2). 
In terms of clinical presentation there are typical and atypical TIa s $(4,5)$ - typical TIa s are characterized by brief, focal neurologic symptoms pertaining to a single cerebrovascular territory (unilateral weakness of the face or limb, unilateral sensory abnormalities, speech transient monocular blindness), whereas atypical TIAs include: gradual build-up of symptoms (for more than minutes) or, by contrast, very short episodes (less than 30 seconds); symptoms being expressed progressively and successively in different parts of the body (without crossing the midline) or isolated disturbance of vision in both eyes characterized by the occurrence of positive phenomena (2).

The TIa represents a neurologic emergency (6) as these patients have a high early risk of developing a subsequent ischemic stroke (7). The diagnosis of TIa is mainly based on the history of the episode as reported by the patient and/or witnesses and the clinical features of the transient attack. a s the very of the TIA pinpoints the absence of structural changes (as opposed to its main counterpart and next stage of the ischemic process - the ischemic stroke) (1), the urgent cerebral and neurovascular imaging studies are part of the initial evaluation. Laboratory tests, electroencephalography and neurocognitive tests are helpful in ruling out metabolic, psychiatric and other cerebral causes of the neurologic symptoms $(5,8,9)$.

The differential diagnosis of the TIa includes a great number of causes for the exhibited transient neurological features, with seizures being among the most important ones (2). This case was chosen to be reported due to its particularities of clinical presentation and diagnosis algorithm.

\section{CASE REPORT}

A 72-year old male patient with history of grade 2 arterial hypertension, type 2 diabetes mellitus, prostate adenoma and paroxysmal atrial

(for which he was taking Acenocoumarol) presented to our Neurology Department for recurrent episodes of paresthesia described as numbness felt in the right upper limb. The patient had begun to experience paresthesia two weeks prior to his admittance, each episode lasting for a few minutes. The rate of the paresthesia episodes gradually worsened such as by the time he presented to our Neurology
Department he experienced them multiple times per day.

Based on the initial patient history, as described by himself, the primary hypothesis was that the episodes the patient was complaining about were epileptic in nature, namely simple focal seizures.

The cerebral computed tomography scan pinpointed the bilateral presence of lacunar strokes in the capsular-lenticular region and of parietal atheromatous lesions of the vertebral arteries and of the intracavernous segment of both internal carotid arteries.

Furthermore, video-electroencephalography was performed, but it revealed no abnormal

With this cornerstone in the diagnosis process, we went back to make a more thorough account of the patient's paresthesia episodes. The latter detailed anamnesis revealed that the paresthesia described as numbness felt in the right upper limb was also felt in the left part of the upper and lower lips. Moreover, the numbness episodes were being associated in an irregular fashion with blurred vision, loss of balance and coordinating his right upper limb. a t this point, the supposed culprit was a repeated transient ischemic attack in the vertebrobasilar territory.

The general physical and the neurology examinations were normal.

The blood tests revealed moderate syndrome, mixed dyslipidemia, an abnormal coagulation (elevated aPTT and INR) and high glycosylated hemoglobin.

Furthermore, in the process of ruling out different pathologies that could have been the stem of the patient's complaints, neurocognitive testing as well cardiology examination were carried out. The patient was diagnosed with mild cognitive impairment associated with major depressive disorder. Subsequently, the cardiology included ischemic cardiomyopathy with left ventricle hypertrophy, decreased left ventricle ejection fraction and mild degenerative mitral valve regurgitation.

The cerebral magnetic resonance imaging scan revealed enlargement of the periencephalic spaces and of the ventricular system due to moderate diffuse cerebral atrophy as well as millimetric T2-weighted/FLAIR hyperintensities located in the supratentorial, paratrigonal, frontal and capsular, 
but also central pontine regions. The magnetic resonance angiography (Fig. 1) showed irregular stenotic narrowing lesions of atheromatous origin in the intracavernous segment of the internal carotid artery, multiple stenotic atheromatous plaques, dilations and rapid irregular blood in the basilar artery, especially in its prepontine segment. Furthermore, the basilar artery exhibited a sinuous aspect with severe enlargement of its lumen and it grooved and displaced the pons with respect to the midline.

The angio-computed tomography scan of the supra-aortic trunks (Fig. 2) showed multiple vascular lesions consisting of hard and soft plaques some of which caused hemodynamically narrowing of the cervical-cerebral arteries: $50 \%$ stenosis in the V4 segment of the right and left vertebral arteries, $60 \%$ stenosis in the intracavernous segment of both internal carotid arteries as well as two stenoses - a $90 \%$ one in the proximal segment and a $80 \%$ one in the middle third part of the basilar artery.

During his stay in our clinic, the patient exhibited another ten minutes long episode with clinical features ranging from left hemifacial and right upper limb paresthesia to dysphonia, dysphagia, right side hemiparesis and right upper limb ataxia.

Taking into consideration the patient history and all of the aforementioned investigations the patient was diagnosed with vertebrobasilar transient ischemic attacks.

The patient started treatment with Clopidogrel associated with non-vitamin $\mathrm{K}$ anticoagulant Dabigatran (switched from Acenocoumarol during hospitalization) and was discharged nine days later with complete resolution of the TIAs.

\section{DISCUSSIONS}

e ven though TIa is a common diagnostic challenge in the neurological (10), it is not with lesser importance, but essentially imperative to pursue emergency investigation in order to identify the cause and establish preventive treatment as to the very high early risk of ischemic stroke (11). There is a $5.2 \%$ risk of ischemic stroke at seven days from the initial TIA, with the highest risk being within the day (12). Nevertheless, $15-30 \%$ of the ischemic strokes are preceded by TIAs (13).
In this respect the $\mathrm{ABCD} 2$ score (which includes age, blood pressure, clinical features, duration and diabetes mellitus) is an important clinical prediction tool used to identify those patients with suspected TIa at high risk of developing early ischemic stroke (7).

The diagnosis of TIA is often in clinical practice as the clinical at the time of as sessment would have already resolved, thus rendering the physician in the position of using and interpreting the details of the event as recalled and described by the patient and/or witnesses (6). According to some studies, non-stroke specialists correctly $\quad 10 \%$ to $49 \%$ of patients as having suffered a TIA and mistook $31 \%$ to $55 \%$ of patients with non-cerebrovascular transient symptoms as having had TIa (7). Depending on the method of diagnosis and the referral pathway almost $60 \%$ of patients who present to the Neurology Department for supposed TIA do not have it as a diagnosis (14).

a $\mathrm{s}$ to the topic of the differential diagnosis, it can be divided into two main pathways: intrinsic and extrinsic. The intrinsic pathway involves rare occurring types of TIa , that through their particular clinical features represent a diagnosis challenge such as: limb-shaking TIa s (rhythmic, involuntary jerky limb movements in hemodynamic TIa s, which may thus be mistaken for focal motor seizures) (15), TIAs with altered conscious level or other brainstem symptoms (thalamic or brainstem 'locked-in' TIa s) (16), spontaneous limb movements or posturing due to brainstem ischemia (rhythmic movements of the limbs sometimes with prolonged tonic muscle contractions in acute brainstem ischemia) (17) and capsular/pontine warning syndrome ischemia and neural dysfunction limited to the internal capsule or the pons due to of the perforating arteries) $(18,19)$.

Frequent causes of transient neurological symptoms that can mimic TIa include: acephalgic migraine aura (up to about $20 \%$ of patients with suspected TIA) (10), seizure, syncope, functional or anxiety related disturbances, cerebral amyloid angiopathy and brief metabolic abnormalities (20).

In addition to electroencephalography and neuroimaging studies, patient history is of utmost sigin distinguishing TIAs from seizures (21). Useful historical features include: epidemiological 
data, type of symptoms ('positive' versus 'negative'), progression pattern, duration of the episode, precipitating factors and associated signs and symptoms during and after the attacks $(2,11)$.

The epidemiological data revealed that TIa $\mathrm{s}$ are rarely reported in young people without vascular risk factors, but when they happen, TIAs are more common in young men, with the sex gap being reduced postmenopausal. at the same time, seizures have no sex or age predilection (22-24).

a $s$ to the nature of the symptoms, they can be categorized as being 'positive' or 'negative'. Positive symptoms indicate the presence of an excessive central nervous system electrical discharges and may be special sensory (e.g. lights, zigzag shapes, tinnitus, music), somatosensory (e.g. pain, paraesthesia) or motor (e.g. jerking limb movements or repetitive rhythmic movements). Negative symptoms indicate a loss or reduction of central nervous system function (e.g. loss of vision, sensation or limb control) (11). TIa s typically begin with negative symptoms, even though over the course of the episode they might develop positive symptoms as well, whereas seizures usually begin with positive symptoms in one modality (25) which progress very quickly during seconds and occasionally cause paralytic attacks. Loss of consciousness is very common in seizures whereas it is extremely rare in TIa s. Regarding the pattern of the attacks - seizures usually cause stereotyped attacks; by comparison, symptoms are more in various TIa s from one episode to another (11). While loss of consciousness is common in seizures it is extremely rare in TIa $\mathrm{s}(9)$.

a $\mathrm{s}$ to the duration of the episodes and their span over periods of time, TIa s usually last less than one weeks). Sometimes TIAs can appear at a more rapid pace, as a cluster, in the so-called 'crescenhour and occur over a short time-frame (days to

do'/'shotgun-like' attacks ( $\geq 3$ TIAs within the preceding seven days) (20,26). Seizures usually last a couple of minutes and are likely to occur during a much larger timespan (years) either sporadi-

Precipitating factors for seizures include hyperventilation, bouts of sepsis and alcool consumption, stroboscopic stimulation, drug withdrawal or anticonvulsant treatment interruption (27). sudden orthostatism, antihypertensive medication, a large meal or a steamy hot bath can trigger hemodynamic "jerking” TIa s $(15,28)$.

a ssociated signs and symptoms such as a bitten tongue (especially if lateral), sphincter incontinence and muscle aches after the event can be postictal markers and vomiting has been reported in both TIa s and seizures. Headache is common following a seizure and ca occur during a TIA, but rarely simultaneously or immediately after neurologic symptoms (9).

\section{CONCLUSIONS}

TIa is a common and important diagnostic challenge in the Neurology Department. In such cases it is paramount to pursue emergency investigation in order to identify the cause and establish preventive treatment as to the very high risk of developing a subsequent ischemic stroke. e ven though patient history (as described by him/herself or by the witnesses of his/her attacks) is fundamental for certain diagnosis, the transience of the symptoms makes certainty challenging. Together with careful clinical examination and imaging studies, effective distinction of TIa from the myriad of differential pathologies is to be achieved.

This case was chosen to be presented in order to highlight another facet of the clinical polymorphism exhibited by transient ischemic attacks.

Conflict of interest: none declared Financial support: none declared

\section{REFERENCES}

1. Easton JD, Saver JL, Albers GW, et al. Definition and Evaluation of Transient Ischemic Attack. Stroke. 2009;40(6):2276-93.

2. Furie KL, Hakan A. Definition, etiology, and clinical manifestations of transient ischemic attack - UpToDate [Internet]. UpToDate. 2018.

3. Kappelle LJ, van Latum JC, van Gijn J, et al. Transient ischemic attacks and small-vessel disease. Lancet. 1991;337(8737):339-41.

4. Fisher $\mathrm{CM}$. Late-life migraine accompaniments - further experience. Stroke. 1986;17(5):1033-42.
5. Tuna M. New insights in the management of patients with ischemic stroke or TIA - Level 2. In: 4rd Congress of the European Academy of Neurology. 2018.

6. Edlow JA. Managing Patients With Transient Ischemic Attack. Ann Emerg Med. 2018;71(3):409-15.

7. Sheehan OC, Merwick A, Kelly LA, et al. Diagnostic usefulness of the $A B C D 2$ score to distinguish transient ischemic attack and minor 
ischemic stroke from noncerebrovascular events: the North Dublin TIA Study. Stroke. 2009;40(11):3449-54.

8. Coutts SB. Diagnosis and Management of Transient Ischemic Attack. Continuum (Minneap Minn). 2017;23(1, Cerebrovascular Disease):82-92.

9. Hankey GJ, Macleod M, Gorelick PB, Chen C, Caprio FZ, Mattle H (eds.). Warlow's Stroke: Practical Management, 4th Edition. Wiley-Blackwell, 2019.

10. Schrock JW, Glasenapp M, Victor A, et al. Variables Associated With Discordance Between Emergency Physician and Neurologist Diagnoses of Transient Ischemic Attacks in the Emergency Department. Ann Emerg Med. 2012;59(1):19-26.

11. Nadarajan V, Perry RJ, Johnson J, et al. Transient ischemic attacks: mimics and chameleons. Pract Neurol. 2014;14(1):23-31.

12. Giles MF, Rothwell PM. Risk of stroke early after transient ischemic attack: a systematic review and meta-analysis. Lancet Neurol. 2007;6(12):1063-72.

13. Giarola BF, Leyden J, Castle S, et al. Transient Ischemic Attack Rarely Precedes Stroke in a Cohort with Low Proportions of Large Artery Atherosclerosis: A Population-Based Study. Cerebrovasc Dis Extra. 2018;8(2):101-5.

14. Prabhakaran S, Silver AJ, Warrior L, et al. Misdiagnosis of Transient Ischemic Attacks in the Emergency Room. Cerebrovasc Dis. 2008;26(6):630-5.

15. Schulz UGR, Rothwell PM. Transient ischemic attacks mimicking focal motor seizures. Postgrad Med J. 2002;78(918):246-7.

16. Special report from the National Institute of Neurological Disorders and Stroke. Classification of cerebrovascular diseases III. Stroke. 1990;21(4):637-76.

17. Wilson LK, Benavente OR, Woolfenden AR, et al. Spontaneous limb movements and posturing secondary to acute basilar artery occlusion: a potentially devastating seizure mimic. Pract Neurol. 2014;14(1):42-4.

18. Donnan GA, O'Malley HM, Quang L, et al. The capsular warning syndrome: pathogenesis and clinical features. Neurology. 1993;43(5):957-62.

19. Enriquez-Marulanda A, Amaya-Gonzalez P, Orozco JL. Pontine Warning Syndrome. Neurologist. 2016;21(6):93-6.

20. Caplan LR. Differential diagnosis of transient ischemic attack and acute stroke - UpToDate [Internet].

21. Amort M, Fluri F, Schäfer J, et al. Transient Ischemic Attack versus Transient Ischemic Attack Mimics: Frequency, Clinical Characteristics and Outcome. Cerebrovasc Dis. 2011;32(1):57-64.

22. Degan D, Ornello R, Tiseo C, et al. Epidemiology of Transient Ischemic Attacks Using Time- or Tissue-Based Definitions: A Population-Based Study. Stroke. 2017;48(3):530-6.

23. American Academy of Neurology. H, Malik A, Thomas A, et al. Neurology. Vol. 84, Neurology. Advanstar Communications; 2015. P2.306.

24. Sirven JI. Epilepsy: A Spectrum Disorder. Cold Spring Harb Perspect Med. 2015;5(9):a022848.

25. Kaplan PW. Focal Seizures Resembling Transient Ischemic Attacks due to Subclinical Ischemia. Cerebrovasc Dis. 1993;3(4):241-3.

26. Karkos CD, McMahon G, McCarthy MJ, et al. The value of urgent carotid surgery for crescendo transient ischemic attacks. J Vasc Surg. 2007;45(6):1148-54.

27. Chowdhury RN, Hasan MH, Rahman KM, et al. Precipitating factor of seizure in epilepsy: experience in a tertiary care hospital. Mymensingh Med J. 2014;23(1):56-61.

28. Das A, Baheti NN. Limb-shaking transient ischemic attack. J Neurosci Rural Pract. 2013;4(1):55-6. 